\title{
Stochastic Properties and Application of City Section Mobility Model
}

\author{
Md. Shohrab Hossain and Mohammed Atiquzzaman \\ Telecommunications and Networks Research Lab \\ School of Computer Science, University of Oklahoma, Norman, OK 73019 \\ Email: $\{$ shohrab, atiq $\} @$ ou.edu
}

\begin{abstract}
Node mobility has a direct impact on the performance evaluation of various network mobility protocols. Unfortunately, most of the analysis on mobility protocols used Random Waypoint mobility model which does not represent real-world movement patterns of mobile nodes. In this paper, we have analyzed City Section mobility model, a realistic mobility model for the movement in the city streets. We have developed an analytical model to derive certain stochastic properties, such as, expected epoch length, expected epoch time, expected number of subnet crossings and subnet residence time of this model. Finally, we have applied the model to calculate the signaling cost of NEMO BSP and compared it with Random Waypoint mobility model. Results show that the use of realistic mobility model leads to better estimation of the signaling cost of network mobility protocol.
\end{abstract}

Index Terms-Mobility Model, Handover, Network Mobility, Performance Evaluation, Mathematical Modeling

\section{INTRODUCTION}

Increasing demand for mobility in wireless data networks has given rise to various mobility management schemes. Network mobility has been extensively studied for the last few years. IETF has proposed NEtwork MObililty Basic Support Protocol (NEMO BSP) [1] to support the mobility of a network. To simulate network mobility, mobility models which try to mimic the movement pattern of mobile networks are used. Mobility should be modeled in a realistic manner, otherwise simulation result may not fit well in a real world deployment. Previous research [2] has also illustrated that the choice of a mobility model can significantly affect the performance evaluation of network protocols. Examples of various mobility models are Random Walk, Random WayPoint (RWP), Random Direction, Gauss-Markov, and City Section. Among these entity mobility models, Random Waypoint model is the most common and frequently used model due to its simplicity. But the movement pattern represented by RWP model is not realistic. It picks a random speed and direction, resulting in sharp turns and sudden stops frequently which is very unusual in real scenarios.

In contrast, City Section (CitySec) mobility model, introduced by Davies [2], [3], [4], represents movement behavior that is influenced by constraints in the environment. In real life scenarios, mobile nodes do not have the ability to travel freely; they have to follow traffic regulations, avoid obstacles,

The research reported in this paper was funded by NASA Grant NNX06AE44G. buildings etc. In fact, City Section mobility model is a realistic movement pattern for vehicles in a city.

There exists some research works on street mobility models in the literature and most of those are simulation based. Martinez et al. [5] develop a simulation tool called CityMob which is a mobility pattern generator to be used with the ns2 simulator. Saha et al. [4] analyze a realistic street mobility model using a real street map; their tool generates scenarios in a format that is compatible with ns-2 format. Jardosh et al. [6] propose a mobility model that enables the inclusion of obstacles to restrict node movement and wireless transmissions in ad hoc network simulations. Haerri et al. [7] present VanetMobiSim, another generator of realistic vehicular movement traces for telecommunication networks simulators. Bettstetter et al. [8] analyze the stochastic properties of the RWP model. Though most of the work on street mobility models are simulation based, they are not analytically tractable. This work differs from previous works in this respect and presents a detailed mathematical analysis of the City Section mobility model.

Our objective in this paper is to develop an analytical model for a realistic mobility model to simulate the movement of a Mobile Network in a section of a city. The mathematical analysis presented in this paper explains analytically how certain parameters of CitySec model can influence its epoch length, epoch time, number of subnet crossings, and subnet residence time, thus giving a deeper understanding of the behavior of the model.

Our contributions in this paper are (i) developing an analytical model to derive equations for expected epoch length, variance of epoch length, mean epoch time, expected number of subnet crossing, and subnet residence time of CitySec model, and (ii) demonstrating the use of the model to compute the signaling cost of NEMO BSP and showing the difference with RWP model.

The rest of the paper is organized as follows. In section II, the CitySec model is described in brief. In section III, various stochastic properties of the model are derived and analyzed. In section IV, we apply the results of CitySec model on NEMO BSP to find out its signaling cost and compare it with RWP model. Finally section $\mathrm{V}$ has the concluding remarks. 


\section{City Section Mobility Model}

The simulation area used in CitySec model [3] is represented by a grid of streets forming a particular section of a city. The model sets the speed limits of each street. Each Mobile Network (MN) begins the simulation at a predefined intersection of two streets. It then randomly chooses a destination, also represented by intersection of two streets. Moving to this destination involves (at most) one horizontal and one vertical movement. Upon reaching the destination, the MN pauses for some random time and the same process is repeated. Each such cycle is termed as an epoch. Following are the assumptions of CitySec model:

- Roads are parallel to axes.

- Starting point and each destination point are assumed to be in road intersections.

\section{Stochastic Properties of CitySec Model}

Let the environment be a rectangular area of dimension $a \times b$ as shown in Fig. 1. Let there be $N_{s}$ horizontal roads (streets) and $N_{a}$ vertical roads (avenues) and streets be $S_{y}$ distance apart and avenues be $S_{x}$ distance apart. So number of streets and number of avenues are

$$
\begin{aligned}
& N_{a}=\frac{a}{S_{x}}+1 \\
& N_{s}=\frac{b}{S_{y}}+1
\end{aligned}
$$

Let us consider that in epoch $i$, MN moves from point $S^{i}$ to point $D^{i}$ via intermediate point $I^{i}$, involving (at most) one horizontal and one vertical movement. So the movement pattern in each epoch of CitySec model can be represented by $\left(S^{i}, V_{x}^{i}, I^{i}, U_{I^{i}}\right),\left(I^{i}, V_{y}^{i}, D^{i}, U_{D^{i}}\right)$. Here, $V_{x}^{i}$ and $V_{y}^{i}$ are speeds of $\mathrm{MN}$ on the two road segments and the pause time at point $P^{i}$ is $U_{P^{i}} \in\left[0, U_{\max }\right]$. The pause times are chosen from a bounded random distribution. In such epoch when the starting point and destination point are on the same (horizontal or vertical) road segment, the intermediate point $I^{i}$ coincides with $D^{i}$. Next, we are going to derive different stochastic properties of City Section mobility model.

\section{A. Epoch Length}

In each epoch, the MN moves along (at most) two road segments. Let $L_{x}^{i}$ and $L_{y}^{i}$ be the lengths of those two road segments during $i$ th epoch. So $L_{x}^{i}=\left|I_{x}^{i}-S_{x}^{i}\right|$ and $L_{y}^{i}=$ $\left|D_{y}^{i}-I_{y}^{i}\right|$. So the total distance covered by the MN during $i$ th epoch is,

$$
L^{i}=L_{x}^{i}+L_{y}^{i}
$$

Therefore, time required for $i$ th epoch is,

$$
t_{i}=\frac{L_{x}^{i}}{v_{x}^{i}}+\frac{L_{y}^{i}}{v_{y}^{i}}+U_{I^{i}}+U_{D^{i}}
$$

Let us number each of the avenues as $1,2, \ldots, N_{a}$ th avenue from left to right and each of the streets as $1,2, \ldots, N_{s}$ th street from top to bottom. The starting point and the destination point of each epoch can only be any two points among these

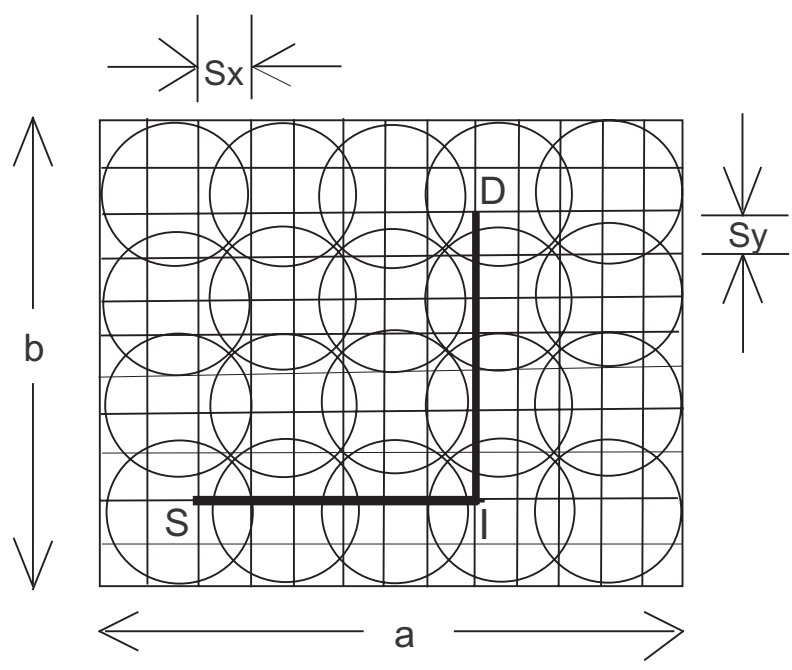

Fig. 1. Road network in City Section mobility model.

$N_{a} N_{s}$ points on the grid. So while considering only horizontal movement path along SI street segment (Fig. 1), both the points $S$ and $I$ can be selected from a street having a maximum of $N_{a}$ discrete values. The probability mass function (pmf) of a randomly selected point's location on a horizontal line of $N_{a}$ discrete points can be given by

$$
f_{P_{x}}(x)= \begin{cases}\frac{1}{N_{a}}, & \text { when } x \in\left\{1,2, \ldots N_{a}\right\} \\ 0, & \text { otherwise }\end{cases}
$$

Since the random selection of these two points are independent of each other, their joint pmf would be

$$
\begin{aligned}
f_{P_{x_{1}}, P_{x_{2}}}\left(x_{1}, x_{2}\right) & =f_{P_{x_{1}}}\left(x_{1}\right) \cdot f_{P_{x_{2}}}\left(x_{2}\right) \\
& = \begin{cases}\frac{1}{N_{a}^{2}}, & \text { when } x_{1}, x_{2} \in\left\{1,2, \ldots N_{a}\right\} \\
0, & \text { otherwise }\end{cases}
\end{aligned}
$$

1) Expected Epoch Length: Let us first find out the expected length that a Mobile Network travels in horizontal direction in an epoch. Let $L_{x}$ be the length of one instance.

$$
L_{x}=\left|P_{x_{1}}-P_{x_{2}}\right|
$$

The values of $L_{x}$ can be found from the $N_{a} \times N_{a}$ matrix below. Each entry of the matrix is given by,

$$
M(i, j)=S_{x}|i-j|, \text { where } 1 \leq i, j \leq N_{a}
$$

$$
\begin{array}{|ccccc|}
0 & S_{x} & 2 S_{x} & . . & \left(N_{a}-1\right) S_{x} \\
S_{x} & 0 & S_{x} & . . & \left(N_{a}-2\right) S_{x} \\
2 S_{x} & S_{x} & 0 & . . & \left(N_{a}-3\right) S_{x} \\
3 S_{x} & 2 S_{x} & S_{x} & . . & \left(N_{a}-4\right) S_{x} \\
\cdot & \cdot & \cdot & . . & . \\
\cdot & \cdot & \cdot & . . & . \\
\left(N_{a}-2\right) S_{x} & \left(N_{a}-3\right) S_{x} & \left(N_{a}-4\right) S_{x} & . . & S_{x} \\
\left(N_{a}-1\right) S_{x} & \left(N_{a}-2\right) S_{x} & \left(N_{a}-3\right) S_{x} & . . & 0
\end{array}
$$

The values of $L_{x}$ can be $0, S_{x}, 2 S_{x}, 3 S_{x}, \ldots,\left(N_{a}-1\right) S_{x}$ depending on the location of points $P_{x_{1}}$ and $P_{x_{2}}$. So the 
expected value of $L_{x}$ is given by,

$$
\begin{aligned}
E\left(L_{x}\right)= & \frac{1}{N_{a}^{2}}\left[N_{a} \times 0+2\left\{\left(N_{a}-1\right) S_{x}+\left(N_{a}-2\right) 2 S_{x}\right.\right. \\
& \left.\left.+. .+2\left(N_{a}-2\right) S_{x}+1\left(N_{a}-1\right) S_{x}\right\}\right] \\
= & \frac{2 S_{x}}{N_{a}^{2}} \sum_{i=1}^{N_{a}-1}\left(N_{a}-i\right) i \\
= & \frac{S_{x}\left(N_{a}^{2}-1\right)}{3 N_{a}}
\end{aligned}
$$

Substituting $a=S_{x}\left(N_{a}-1\right)$, we get

$$
E\left(L_{x}\right)=\frac{a\left(N_{a}+1\right)}{3 N_{a}}
$$

Similarly, for vertical movement,

$$
E\left(L_{y}\right)=\frac{b\left(N_{s}+1\right)}{3 N_{s}}
$$

Now adding Eqns. (8) and (9), the expected epoch length can be obtained.

$$
E(L)=\frac{a\left(N_{a}+1\right)}{3 N_{a}}+\frac{b\left(N_{s}+1\right)}{3 N_{s}}
$$

In Fig. 2, the expected epoch length of CitySec model is compared with that of RWP Model [8]. Here, $E(L) / a$ is plotted against $b / a$ for different number of avenues for CitySec model using Eqn. (10); number of streets $\left(N_{s}\right)$ is obtained by $N_{s} \approx(b / a) N_{a}$ (see Eqns. (1) and (2)). It is found that expected epoch length of CitySec mobility model is much higher than that of RWP mobility model irrespective of the value of $N_{a}$. For large values of $N_{a}$ and $N_{s}$, Eqn. (10) reduces to

$$
E(L)=\frac{a}{3}+\frac{b}{3}
$$

For a square grid of size $a \times a, E(L)=2 a / 3$. This expected epoch length of City Section mobility model gives a measure of the distance covered by a mobile node in an epoch on the average which is required while analyzing mobility and handover protocols.

2) Variance of Epoch Length: The variance of epoch length can tell us how the epoch lengths vary about its mean value and gives some indication of the randomness of the CitySec mobility model. First, the second moment of $L_{x}$ is obtained by,

$$
\begin{aligned}
E\left(L_{x}^{2}\right)= & \frac{1}{N_{a}^{2}}\left[N_{a}\left(0^{2}\right)+2\left\{\left(N_{a}-1\right) S_{x}^{2}+\left(N_{a}-2\right)\left(2 S_{x}\right)^{2}\right.\right. \\
& \left.\left.+. .+2\left\{\left(N_{a}-2\right) S_{x}\right\}^{2}+1\left\{\left(N_{a}-1\right) S_{x}\right\}^{2}\right\}\right] \\
= & \frac{2 S_{x}^{2}}{N_{a}^{2}} \sum_{i=1}^{N_{a}-1}\left(N_{a}-i\right) i^{2} \\
= & \frac{S_{x}^{2}\left(N_{a}^{2}-1\right)}{6}
\end{aligned}
$$

Substituting $a=S_{x}\left(N_{a}-1\right)$, we get

$$
E\left(L_{x}^{2}\right)=\frac{S_{x}\left(N_{a}+1\right) a}{6}
$$

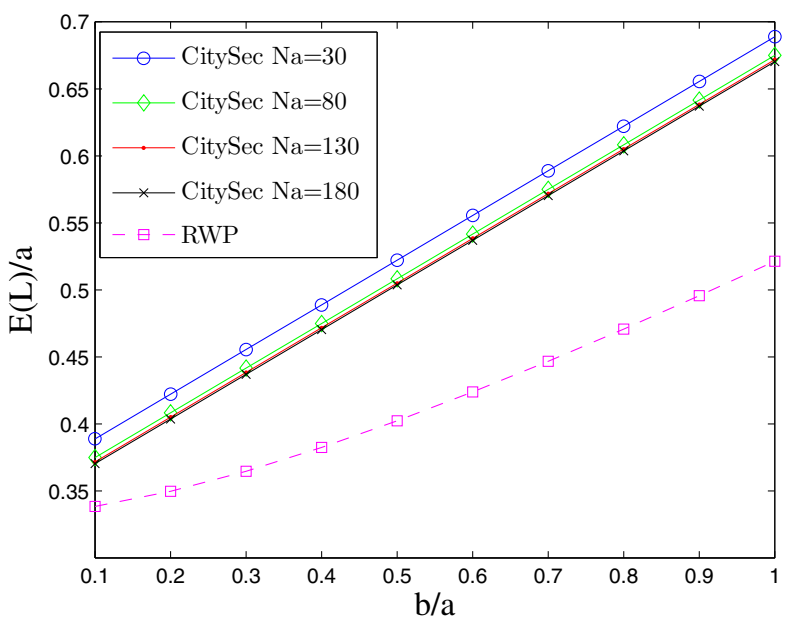

Fig. 2. Expected epoch length of City Section and RWP mobility model within $a \times b$ rectangle.

Similarly,

$$
E\left(L_{y}^{2}\right)=\frac{S_{y}\left(N_{s}+1\right) b}{6}
$$

The second moment of $L$ is therefore given by,

$$
E\left(L^{2}\right)=\frac{S_{x}\left(N_{a}+1\right) a}{6}+\frac{S_{y}\left(N_{s}+1\right) b}{6}
$$

Now the variance of $L_{x}$ can be obtained by,

$$
\begin{aligned}
V\left(L_{x}\right) & =E\left(L_{x}^{2}\right)-\left(E\left(L_{x}\right)\right)^{2} \\
& =\frac{S_{x}^{2}\left(N_{a}^{2}-1\right)}{3}\left[\frac{1}{2}-\frac{\left(N_{a}^{2}-1\right)}{3 N_{a}^{2}}\right]
\end{aligned}
$$

For large $N_{a}, N_{a}^{2}-1 \approx N_{a}^{2}$. So

$$
V\left(L_{x}\right)=\frac{S_{x}^{2}\left(N_{a}^{2}-1\right)}{18}=\frac{E\left(L_{x}^{2}\right)}{3}
$$

Similarly,

$$
V\left(L_{y}\right)=\frac{S_{y}^{2}\left(N_{s}^{2}-1\right)}{18}=\frac{E\left(L_{y}^{2}\right)}{3}
$$

Therefore, the variance of the epoch length is given by,

$$
V(L)=\frac{S_{x}^{2}\left(N_{a}^{2}-1\right)}{18}+\frac{S_{y}^{2}\left(N_{s}^{2}-1\right)}{18}
$$

\section{B. Epoch Time}

The next step is to find out the duration of each epoch using the result of epoch length as follows:

$$
T=\frac{1}{v} L
$$

Let the speed of the MN vary between $V_{x}^{\text {min }}$ to $V_{x}^{\max }$ for some horizontal road segment. So for uniform speed distribution, the probability density function of MN's speed in horizontal direction can be given by

$$
f_{V}\left(v_{x}\right)=\frac{1}{V_{x}^{\max }-V_{x}^{\min }}
$$




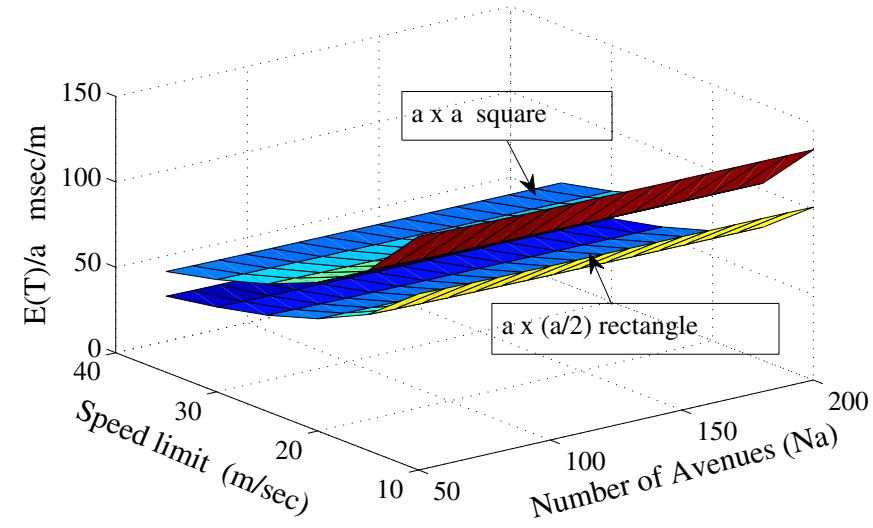

Fig. 3. Expected epoch time of CitySec mobility model as a function of speed limit and number of avenues (streets).

The expected time required for movement on horizontal road segment in an epoch is thus,

$$
\begin{array}{r}
E\left(T_{x}\right)=E\left(L_{x}\right) \int_{V_{x}^{\min }}^{V_{x}^{\max }} \frac{1}{v_{x}} f_{V}\left(v_{x}\right) d v_{x} \\
=E\left(L_{x}\right) \frac{\ln \left(V_{x}^{\max } / V_{x}^{\text {min }}\right)}{V_{x}^{\text {max }}-V_{x}^{\text {min }}}
\end{array}
$$

Similarly, for movement on vertical road segment

$$
E\left(T_{y}\right)=E\left(L_{y}\right) \frac{\ln \left(V_{y}^{\max } / V_{y}^{\min }\right)}{V_{y}^{\max }-V_{y}^{\text {min }}}
$$

Adding Eqns. (20) and (21), the expected epoch time can be found as,

$$
\begin{aligned}
E(T)= & \frac{a\left(N_{a}+1\right) \ln \left(V_{x}^{\max } / V_{x}^{\text {min }}\right)}{3 N_{a}\left(V_{x}^{\max }-V_{x}^{\text {min }}\right)} \\
& +\frac{b\left(N_{s}+1\right) \ln \left(V_{y}^{\text {max }} / V_{y}^{\text {min }}\right)}{3 N_{s}\left(V_{y}^{\text {max }}-V_{y}^{\text {min }}\right)}
\end{aligned}
$$

In Fig. 3, the expected epoch time of City Section mobility model is shown for $a \times a$ square and $a \times(a / 2)$ rectangular road networks. Here, we assumed $V_{x}^{\text {min }}=V_{y}^{\text {min }}=2 \mathrm{~m} / \mathrm{sec}$, and $V_{x}^{\max }=V_{y}^{\max }=V^{\max }$. We find that the expected epoch time decreases with the increase of $V^{\max }$, which is quite obvious. On the other hand, expected epoch time increases with the increase in number of avenues $\left(N_{a}\right)$ and streets $\left(N_{s}\right)$. Higher value of $N_{a}$ means larger grid dimensions for fixed inter-road spacing (see Eqns. (1) and (2)), resulting in higher epoch time.

\section{Pause Time}

For safety at road intersections, there is random pause time between 0 to $U_{\max }$ in order to avoid collisions. The expected pause time of the $\mathrm{MN}$ at each intersection of its path can be given by,

$$
E(U)=\int_{0}^{U_{\max }} \frac{u d u}{U_{\max }}=\frac{U_{\max }}{2}
$$

\section{Number of Subnet Crossing}

Let us consider that the road network of dimensions $a \times b$ (Fig. 1) is covered by Access Points (AP); let there be $n$ rows of APs and $m$ APs in each row. In total, there will be $m n$ APs to cover the rectangular area. Let the radio coverage area of each AP be a circular region of radius $r$ and two successive APs overlap at a maximum length of $l$ along its diameter. So

$$
\begin{gathered}
a=2 r m-(m-1) l \\
b=2 r n-(n-1) l
\end{gathered}
$$

Let the radius $r$ of each subnet be greater than the spacing between successive road segments, i.e., $r>S_{x}$ and $r>S_{y}$. Let us assume that $2 r=K S_{x}=K S_{y}$ and $l=S_{x} / 2=S_{y} / 2$. Now from Eqn. (1), we get $a=\left(N_{a}-1\right) S_{x}$. Equating this value of $a$ with Eqn. (24), we get,

$$
m=\left\lceil\frac{2 N_{a}-3}{2 K-1}\right\rceil
$$

Similarly, we can have

$$
n=\left\lceil\frac{2 N_{s}-3}{2 K-1}\right\rceil
$$

Let us first consider the movement along horizontal direction. If the distance between the two endpoints is less than the diameter of an AP's coverage area i.e., between 0 to $(K-1) S_{x}$, there will be at most one subnet crossing. For any distance between $K S_{x}$ to $(2 K-1) S_{x}$, there will be at most two subnet crossings, and so on. Thus, if the point $S$ is at first avenue and point $I$ is at $N_{a}$ th avenue, then the distance of the road segment will be $\left(N_{a}-1\right) S_{x}$ and there will be at most $m$ subnet crossings. Thus we can find out the expected number of subnet crossings in an epoch for movement along horizontal direction as,

$$
\begin{aligned}
E\left(C_{x}\right) & =\frac{2}{N_{a}^{2}}\left[\left(N_{a}-1\right)+\left(N_{a}-2\right)+. .+N_{a}-(K-1)+\right. \\
2 & \left\{\left(N_{a}-K\right)+\left(N_{a}-K-1\right)+. .+\left(N_{a}-2 K+1\right)\right\} \\
& +. .+m\left\{\left(N_{a}-(m-1) K\right)+\left(N_{a}-(m-1) K-1\right)\right. \\
& \left.\left.+. .+\left(N_{a}-m K+1\right)\right\}\right] \\
& =\frac{2}{N_{a}^{2}}\left[K N_{a} \sum_{i=1}^{m} i-K^{2} \sum_{i=2}^{m} i(i-1)-\sum_{i=1}^{K-1} i \sum_{j=1}^{m} j\right] \\
& =\frac{m(m+1) K}{6 N_{a}{ }^{2}}\left(6 N_{a}-4 m K+K+3\right)
\end{aligned}
$$

Similarly, for movement along vertical direction,

$$
E\left(C_{y}\right)=\frac{n(n+1) K}{6 N_{s}^{2}}\left(6 N_{s}-4 n K+K+3\right)
$$

The expected number of subnet crossing in an epoch is thus

$$
E(C)=E\left(C_{x}\right)+E\left(C_{y}\right)
$$




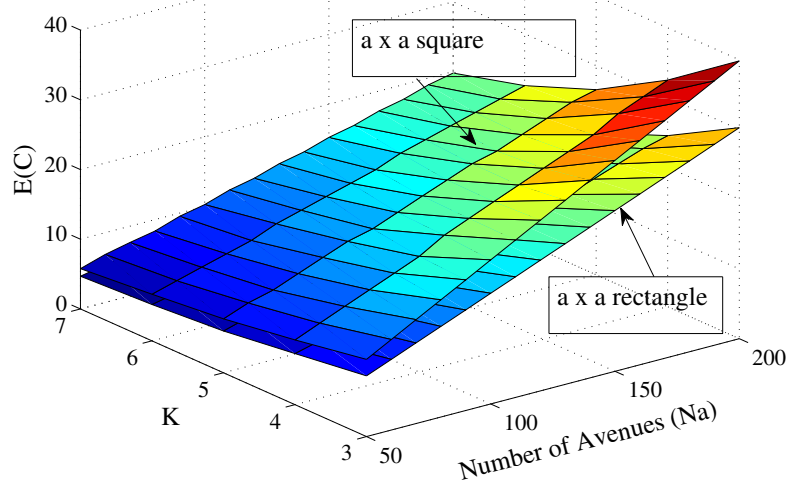

Fig. 4. Expected number of subnet crossings of City Section mobility model as a function of $\mathrm{K}$ and number of avenues.

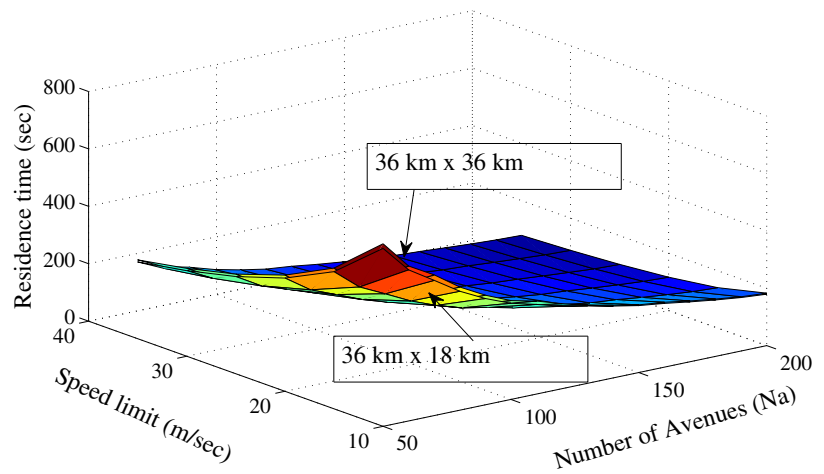

Fig. 5. Residence time of City Section mobility model as a function of speed limit and number of avenues.

In Fig. 4, the expected number of subnet crossings of CitySec mobility model is shown for $a \times a$ square and $a \times(a / 2)$ rectangular road networks. We find that the expected number of subnet crossings decreases with the increase in the value of $K$ (i.e., $2 r / S_{x}$ ). Higher values of $K$ mean larger coverage area for access points, resulting in fewer number of subnet crossings. On the other hand, expected number of subnet crossings increases with the increase in number of avenues $\left(N_{a}\right)$ and streets $\left(N_{s}\right)$. Higher values of $N_{a}$ and $N_{s}$ mean larger grid dimensions for fixed inter-road spacing (see Eqns. (1) and (2)), resulting in higher number of subnet crossings.

\section{E. Subnet Residence Time}

Since in each epoch, the MN pauses at two different points, the average residence time of a $\mathrm{MN}$ in a subnet can be estimated as follows:

$$
T_{r}=\frac{E(T)+2 E(U)}{E(C)}
$$

In Fig. 5, the residence time of City Section mobility model is shown for $36 \mathrm{~km} \times 36 \mathrm{~km}$ square and $36 \mathrm{~km} \times 18$ $\mathrm{km}$ rectangular road networks. Here we used $U_{\max }=2 \mathrm{sec}$, $V_{x}^{\min }=V_{y}^{\min }=2 \mathrm{~m} / \mathrm{s}, K=5$. We find that the residence time decreases with the increase of speed limit as less time is required to cross the coverage area of an AP. Residence time also decreases with the increase in number of avenues $\left(N_{a}\right)$ and streets $\left(N_{s}\right)$. Higher values of $N_{a}\left(N_{s}\right)$ result in increased $E(C)$ values, but the value of $E(T)$ is not affected much. As a result, the residence time decreases (see Eqn. (30)).

\section{Signaling Cost of NEMO BSP}

To demonstrate the application of the developed model, we use the results of CitySec mobility model to compute the signaling cost of NEMO BSP. We consider a mobile network having a number of mobile hosts $\left(N_{m h}\right)$ moving together and being managed by NEMO BSP. Previous work [9] has derived the signaling costs of NEMO BSP which consists of two components: location update cost $\left(\Psi^{L U}\right)$ and lookup cost $\left(\Psi^{L U P}\right)$. Since the lookup cost does not vary for different mobility models, we consider only the location update (LU) cost which is given by,

$$
\begin{array}{r}
\Psi^{L U}=\left[N_{m h}\left\{2\left(l_{m h}-2+2 \theta\right) \delta_{L U}+\gamma_{h}\right\}\right. \\
\left.+N_{m r}\left\{2\left(l_{m r}-1+\theta\right) \delta_{L U}+\gamma_{r}\right\}\right] / T_{r}
\end{array}
$$

Here, $N_{m r}=$ number of mobile routers (MR),

$T_{r}=$ residence time for $\mathrm{MR} / \mathrm{MH}$

$N_{c n}=$ average number of CN communicating,

$\delta_{L U}=$ per hop message transmission cost for LU,

$\theta=$ proportionality constant of signaling cost over wired and wireless link,

$l_{m h}=$ average number of hops between $\mathrm{MH}$ and $\mathrm{HA}-\mathrm{H}$,

$l_{m r}=$ average number of hops between MH and HA-M,

$\gamma_{h}=$ update processing cost at HA-H,

$\gamma_{r}=$ update processing cost at HA-M,

The comparison with RWP model is based on the analysis presented in [8] which assumes that the coverage area of each $\mathrm{AP}$ is a square that is not realistic. For the sake of comparison, we are assuming the dimensions of each AP's coverage area of RWP model to be $2 r \times 2 r$. The parameters relating to NEMO BSP have been taken from the previous works [9], [10]: $\delta_{L U}=$ $0.2, \theta=10, \gamma_{h}=30, \gamma_{r}=1.5 \times \gamma_{h}, l_{m h}=l_{m r}=35$, and $N_{m r}=2$. Other parameters values relating to mobility models are: $a=36 \mathrm{~km}, b=24 \mathrm{~km}, S_{x}=200 \mathrm{~m}, S_{y}=200 \mathrm{~m}, K=5$, $V_{x}^{\text {max }}=V_{y}^{\text {max }}=30 \mathrm{~m} / \mathrm{s}, V_{x}^{\text {min }}=V_{y}^{\text {min }}=5 \mathrm{~m} / \mathrm{s}$, and $U_{\max }$ $=4 \mathrm{sec}$.

Fig. 6 shows the impact of the number of mobile hosts on location update cost of NEMO BSP for both City Section and RWP mobility models. Results show that the location update cost of City Section model is much less and different than that of RWP model.

Fig. 7 shows the subnet residence times of RWP and CitySec model for varying speed of the mobile network. It is found that subnet residence time of City Section mobility model varies a lot with that of RWP mobility model.

Fig. 8 shows the location update cost for NEMO BSP using RWP and CitySec model varying speed of MN for various 


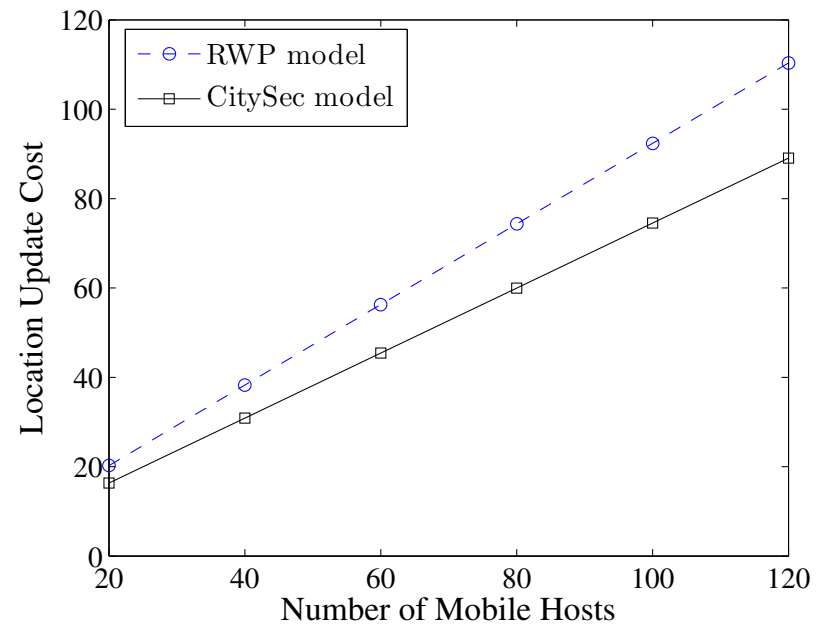

Fig. 6. Location update cost of NEMO BSP vs. number of MHs for CitySec and RWP model.

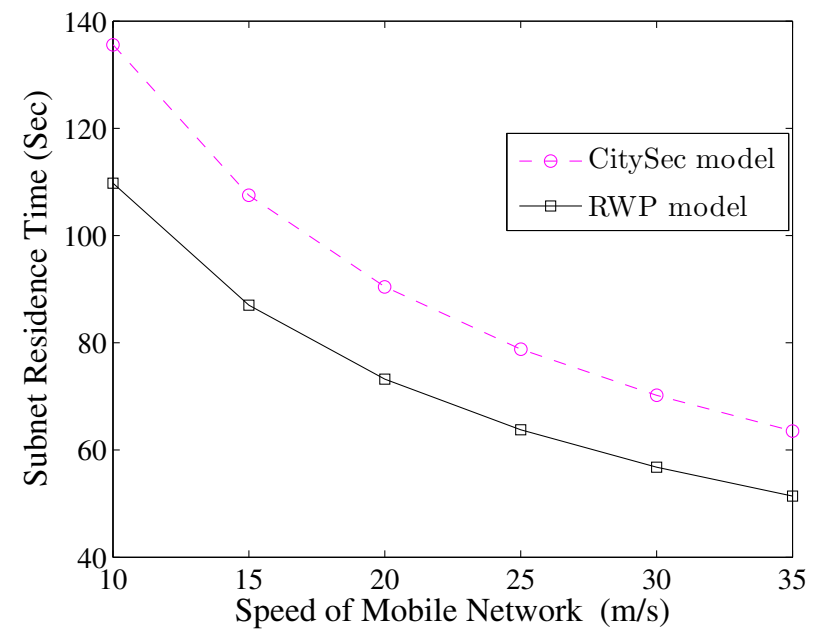

Fig. 7. Subnet residence times of RWP and CitySec models vs. speed of mobile networks.

number of mobile routers. Here also the signaling costs of CitySec model differs much with that of RWP model.

From Figs. 6 - 8, we see that the results obtained from the City Section model for NEMO BSP are different than those obtained from RWP model. Consequently, RWP model cannot be used as an approximation to a realistic model, like City Section mobility model.

\section{CONClusion}

In this paper, we have analyzed City Section mobility model, a realistic model to obtain its stochastic properties. We have derived the expressions for expected epoch length, variance of epoch length, mean epoch time, mean subnet crossings and subnet residence time. These results help in understanding the behavior of CitySec mobility more precisely. We have used the derived stochastic properties for the performance

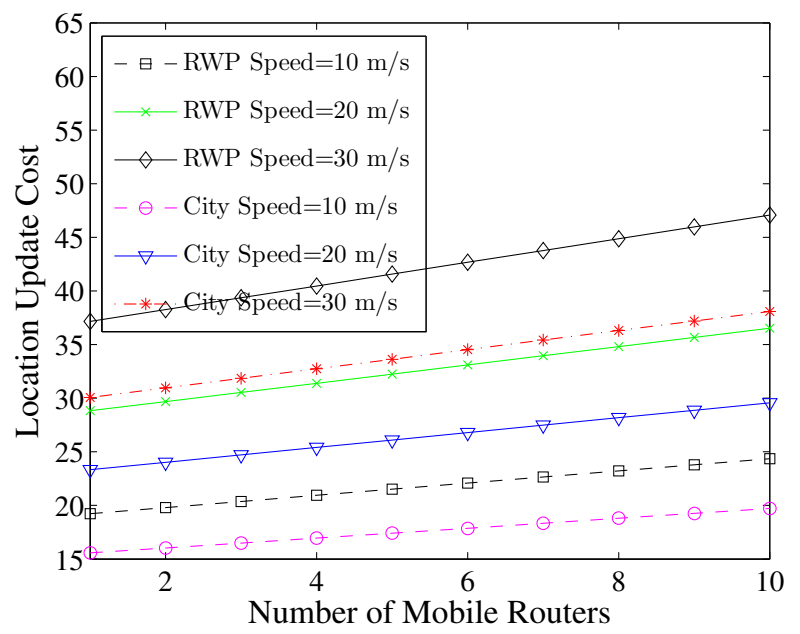

Fig. 8. Location update cost of NEMO BSP vs. Number of Mobile Routers for varying $\mathrm{MN}$ speed.

analysis of of NEMO BSP and compared the results with those using RWP mobility model. Results show that the signaling requirements for these two models are much different. Hence, RWP mobility model cannot be even used as an approximation to a realistic scenario unlike City Section mobility model.

\section{REFERENCES}

[1] V. Devarapalli, R. Wakikawa, A. Petrescu, and P. Thubert, "NEtwork MObility (NEMO) basic support protocol," RFC 3963, Jan 2005.

[2] T. Camp, J. Boleng, and V. Davies, "A survey of mobility models for ad hoc network research," Wireless Communications and Mobile Computing, vol. 2, pp. 483-502, 2002.

[3] V. Davies, "Evaluating mobility models within an Ad Hoc Network," MS thesis, Colorado School of Mines, 2000.

[4] A. K. Saha and D. B. Johnson, "Modeling mobility for vehicular ad hoc networks," in Proceedings of the First ACM International Workshop on Vehicular Ad Hoc Networks (VANET 2004), Philadelphia, PA, Oct. 2004, pp. 91-92.

[5] F. J. Martinez, J.-C. Cano, C. T. Calafate, and P. Manzoni, "Citymob: A mobility model pattern generator for VANETs," in Proceedings of IEEE International Conference on Communications Workshops (ICC 2008), Beijing, China, May 2008, pp. 370-374.

[6] A. Jardosh, E. M. Belding-Royer, K. C. Almeroth, and S. Suri, "Towards realistic mobility models for mobile ad hoc networks," in Proceedings of the Annual International Conference on Mobile Computing and Networking (MOBICOM), San Diego, CA, Sept. 14-19 2003, pp. 217229.

[7] J. Harri, F. Filali, C. Bonnet, and M. Fiore, "VanetMobiSim: Generating realistic mobility patterns for VANETs," in Proceedings of the Third ACM International Workshop on Vehicular Ad Hoc Networks (VANET 2006), Los Angeles, CA, Sep. 29 2006, pp. 96-97.

[8] C. Bettstetter, H. Hartenstein, and X. Prez-Costa, "Stochastic properties of Random Waypoint mobility model," Wireless Networks, vol. 10, no. 5, pp. 555-567, Sept. 2004.

[9] A. S. Reaz, P. K. Chowdhury, and M. Atiquzzaman, "Signaling cost analysis of SINEMO: Seamless End-to-End Network Mobility," in Proceedings of First ACM/IEEE International Workshop on Mobility in the Evolving Internet Architecture, San Francisco, CA, Dec 01, 2006, pp. $37-42$.

[10] J. Xie and I. Akyildiz, "An optimal location management scheme for minimizing signaling cost in Mobile IP," in IEEE International Conference on Communications (ICC), NY, April 2002, pp. 3313-3317. 